\title{
The elephant in the room: the importance of the details of massive star formation in molecular clouds
}

\author{
Michael Y. Grudić ${ }^{\circledR \star}$ and Philip F. Hopkins \\ TAPIR, Mailcode 350-17, California Institute of Technology, Pasadena, CA 91125, USA
}

Accepted 2019 July 1. Received 2019 June 5; in original form 2018 September 21

\begin{abstract}
Most simulations of galaxies and massive giant molecular clouds (GMCs) cannot explicitly resolve the formation (or predict the main-sequence masses) of individual stars. So they must use some prescription for the amount of feedback from an assumed population of massive stars (e.g. sampling the initial mass function, IMF). We perform a methods study of simulations of a star-forming GMC with stellar feedback from UV radiation, varying only the prescription for determining the luminosity of each stellar mass element formed (according to different IMF sampling schemes). We show that different prescriptions can lead to widely varying (factor of $\sim 3$ ) star formation efficiencies (on GMC scales) even though the average massto-light ratios agree. Discreteness of sources is important: radiative feedback from fewer, more-luminous sources has a greater effect for a given total luminosity. These differences can dominate over other, more widely recognized differences between similar literature GMCscale studies (e.g. numerical methods, cloud initial conditions, presence of magnetic fields). Moreover the differences in these methods are not purely numerical: some make different implicit assumptions about the nature of massive star formation, and this remains deeply uncertain in star formation theory.
\end{abstract}

Key words: stars: formation-stars: massive-ISM: clouds - galaxies: formation.

\section{INTRODUCTION}

Massive stars are rare, but their radiation, winds, and supernova explosions dominate the energy liberated from a stellar population. It is thought that feedback from massive stars is a crucial element for regulating star formation on scales ranging from entire galaxies to individual star clusters (McKee \& Ostriker 2007; Naab \& Ostriker 2017). In the latter case, significant theoretical efforts have been devoted to understanding how feedback from massive stars sets the star formation efficiency (SFE) of star-forming giant molecular clouds (GMCs), the fraction of the initial gas mass that is converted to stars before feedback disrupts the cloud and halts star formation. An understanding of the SFE of GMCs is important for understanding the origins of the star cluster mass function and its connection to the GMC mass function (Elmegreen \& Efremov 1997; Fall, Krumholz \& Matzner 2010), the origins of gravitationally bound globular clusters (Hills 1980; Baumgardt \& Kroupa 2007; Kruijssen 2012), and the distribution and pre-conditioning of supernova explosions, which affects the efficiency of stellar feedback on galactic scales (Keller et al. 2014; Fielding, Quataert \& Martizzi 2018).

\footnotetext{
^E-mail: mgrudich@caltech.edu
}

Significant progress has been made on this problem as the necessary computational techniques have become available. Many numerical experiments have been performed in which a selfgravitating molecular cloud is evolved in isolation, subject to selfgravity, hydrodynamics, stellar feedback, and possibly detailed cooling and chemistry physics (Murray, Quataert \& Thompson 2010; Vázquez-Semadeni et al. 2010; Dale, Ercolano \& Bonnell 2012; Colín, Vázquez-Semadeni \& Gómez 2013; Dale et al. 2013; Dale et al. 2014; Skinner \& Ostriker 2015; Howard, Pudritz \& Harris 2016, 2017; Raskutti, Ostriker \& Skinner 2016; Dale 2017; Gavagnin et al. 2017; Kim et al. 2017; Vázquez-Semadeni, González-Samaniego \& Colín 2017; Grudić et al. 2018a; Kim, Kim \& Ostriker 2018b, for review see Krumholz et al. 2014; Dale 2015). For GMC properties consistent with those found in the local Universe $\left(\Sigma_{\text {gas }} \sim 50 \mathrm{M}_{\odot} \mathrm{pc}^{-2}, M \sim 10^{4}-10^{6} \mathrm{M}_{\odot}\right.$, Bolatto et al. 2008), the most important feedback mechanism for regulating star formation on GMC scales is generally agreed to be UV photons from massive stars. UV photons heat and ionize the interstellar medium (ISM) upon absorption by gas or dust, while also imparting momentum upon absorption, creating expanding $\mathrm{H}$ II regions that ultimately unbind the remaining gas in the cloud. However, theoretical consensus on the specific SFE at which cloud disruption occurs (or even whether it occurs at all, Howard et al. 2016) has been slower to develop. As an extreme example, Raskutti et al. (2016) 
simulated a molecular cloud of initial mass $5 \times 10^{4} \mathrm{M}_{\odot}$ and radius $15 \mathrm{pc}$ and obtained an SFE of $\sim 40$ per cent, while Grudić et al. (2018a) simulated the same cloud model with nominally the same feedback physics and obtained $\sim 4$ per cent, an order of magnitude smaller.

Discrepancies are not necessarily surprising when one considers the compounded variations that can arise when using different hydrodynamics methods, sink particle prescriptions, and perhaps most importantly, radiative transfer approximations. Variations due to these numerical details warrant some exploration, as studies that compare radiative transfer methods while controlling for other factors are few, and none are exhaustive. Raskutti et al. (2016) performed simulations treating the effects of photon momentum (i.e. radiation pressure) from UV photon absorption with an M1 radiation transfer scheme (Skinner \& Ostriker 2013), which Kim et al. (2017) subsequently compared to adaptive ray tracing results using otherwise the same code, and found an SFE a factor of $\sim 2$ smaller. Hopkins \& Grudić (2019) also performed GMC simulations comparing the ray-based LEBRON radiative transfer approximation (Hopkins et al. 2018) with an M1 scheme (Rosdahl \& Teyssier 2015), and also found agreement at the factor of $\sim 2$ level. Therefore, variations in radiative transfer techniques can likely account for some of the variation found in the literature, but probably not all of it. This motivates the consideration of other factors.

Several of the studies cited above compared additional physics (e.g. including or ignoring magnetic fields), or varying the cloud initial conditions (e.g. considering clouds with or without preinitialized fully developed turbulence, with or without significant rotational support, and with or without a global density profile): the general conclusion is that these, too, can influence the predicted SFE by at most a factor $\sim 2$ (see references above and Klessen, Heitsch \& Mac Low 2000; Price \& Bate 2008; Krumholz, Klein \& McKee 2011). Others have shown that including or excluding other sources of feedback besides UV radiation alone, e.g. O/B stellar winds (which carry a similar momentum flux to the UV radiation field), can have a similar effect.

In Hopkins \& Grudić (2019) we argued that another potential error source can arise when using the most common method for coupling radiation pressure to gas, which underestimates the imparted momentum from a point source if the photon mean free path is smaller than the fluid resolution. Krumholz (2018) subsequently pointed out another previously overlooked numerical pitfall: photon absorption around an accreting massive star can occur deep in the potential well on scales smaller than the resolution limit of most simulations, preventing it from imparting momentum on larger scales. They argued that the failure to resolve this effect could also explain some of the discrepancy, and proposed a subgrid model to approximate this effect in numerical simulations.

This led us to consider the broader important question that we will address here: how do the details of how the sources of stellar feedback are modelled in simulations affect the cloud-scale SFE? Clearly, when simulations attempt to model the formation of massive stars self-consistently, the details of the initial mass function (IMF) will become important for feedback, as UV luminosity is a steep function of stellar mass. However, most GMC-scale and all galaxy-scale hydrodynamics simulations either lack the resolution or the physical realism to do this self-consistently, so feedback is often treated with phenomenological prescription, assuming an underlying stellar mass distribution that is being sampled in some manner. In this work we will compare several such techniques, and determine the effect of these numerical choices upon the cloud-scale SFE in simulations.

\section{SIMULATIONS}

\subsection{Numerical methods}

We simulate an isolated turbulent molecular cloud with GIZMO, a multiphysics $N$-body and hydrodynamics code (Hopkins 2015). ${ }^{1}$ We solve the equations of hydrodynamics with the Lagrangian Meshless Finite Mass (MFM) method. We account for a wide range of ISM heating and cooling physics, using the rates and implementations used in the FIRE-2 simulations (Hopkins et al. 2018). ${ }^{2}$ Star formation is treated with an accreting sink particle method described in Guszejnov et al. (2018), which uses multiple checks for sink formation and accretion, similar to Federrath et al. (2010). For simplicity, we consider only the effects of feedback due to the absorption of UV photons from stars, accounting for the effects of photoheating and radiation pressure as in Hopkins et al. (2018).

\subsection{Initial conditions}

We replicate the initial conditions of the fiducial cloud model in Kim et al. (2018b), a GMC with a top-hat density profile with mass $10^{5} \mathrm{M}_{\odot}$ and radius $20 \mathrm{pc}$. The initial velocity field is a solenoidal Gaussian random field with power spectrum $|\tilde{\mathbf{v}}(\mathbf{k})|^{2} \propto k^{-4}$ (Gammie \& Ostriker 1996), normalized so that the initial kinetic energy is equal to the gravitational potential energy. The initial metallicity of the cloud is normalized to solar abundances, accounted for self-consistently in the cooling function and the dust opacity to UV photons as in Hopkins et al. (2018). In all simulations we resolve the initial gas mass in $128^{3}$ Lagrangian gas cells, for a mass resolution of $0.048 \mathrm{M}_{\odot}$. Initial conditions were generated with the MAKECLOUD code. ${ }^{3}$

\subsection{IMF sampling models}

We perform simulations with a range of different prescriptions for the specific bolometric and ionizing luminosities assigned to the stellar mass elements (i.e. sink particles) in the simulation. These are all intended to mimic certain aspects of the effect of sampling a finite number of stars from an underlying probability distribution function (i.e. the IMF). Each recovers the same net specific luminosities in the limit $M_{\star}>>10^{3}$, but each approaches that limit in a different manner as stars form in the simulation. All of these prescriptions have advantages and disadvantages - in this work we remain agnostic about the relative physical realism of these models, which is difficult to evaluate without a self-consistent treatment of the physics of massive star formation. We consider only models that work under the assumption that the IMF can indeed be interpreted as probability distribution to be sampled from until a given stellar mass reservoir is exhausted. This must break down at some level, due to mass conservation if no other reason. However, the details of how the IMF emerges are poorly understood, and the stochastic sampling hypothesis is consistent with current observations (Bastian, Covey \& Meyer 2010; Fumagalli, da Silva \& Krumholz 2011; Offner et al. 2014).

\footnotetext{
${ }^{1}$ http://www.tapir.caltech.edu/ phopkins/Site/GIZMO.html

${ }^{2}$ https://fire.northwestern.edu/

${ }^{3}$ https://github.com/omgspace/MakeCloud
} 


\subsubsection{IMFMEAN: simple IMF-averaging}

The simplest approach is to assume that all stellar mass elements in the simulation have the same specific luminosity as a well-sampled IMF, which for a very young stellar population with age $\ll 3.5 \mathrm{Myr}$ and a Kroupa (2002) IMF is approximately

$$
\left\langle\frac{L_{\star}}{M_{\star}}\right\rangle_{\mathrm{IMF}}=1140 L_{\odot} \mathrm{M}_{\odot}^{-1} .
$$

This is approximately constant until $t \approx 3.5 \mathrm{Myr}$, then decreases appropriately as massive stars die according to an adopted stellar evolution model (e.g. Leitherer et al. 1999). The well-sampled assumption is expected to be valid in systems where the total stellar mass is $>10^{3} \mathrm{M}_{\odot}$, and is a common choice for galaxy simulations that might not even resolve mass scales smaller than this (Hopkins, Quataert \& Murray 2011; Agertz et al. 2013; Hopkins et al. 2018), although it has also been used in smaller scale cluster formation simulations (Grudić et al. 2018a,b; Kim et al. 2018a; Hopkins \& Grudić 2019). This is the only prescription that guarantees that the specific luminosity is always equal to the ensemble over all possible IMF samplings. However, this is not necessarily desirable in all problems. The method has a serious drawback in the regime of low-mass star cluster formation: when sampling an IMF from a small reservoir of stellar mass, most realizations sample no massive stars at all. Therefore, the mean specific luminosity is due to those very few possible samplings that do contain massive stars and have specific luminosities much greater than the mean. The effect of this is to give a specific luminosity that is much larger than the vast majority of possible realizations of low-mass clusters, and much less than those few realizations that do, averaging out a major source of physically real stochasticity.

In addition to the standard IMFMEAN scheme, we consider a variant supplemented by the subgrid model introduced by Krumholz (2018), IMFMEAN-K18. To mimic the effect of photon absorption in a dust destruction front at unresolved scales, we simply switch off UV feedback from a sink particle when its accretion rate exceeds the threshold value

$$
\frac{\dot{M}}{\mathrm{M}_{\odot} \mathrm{yr}^{-1}}>6.5 \times 10^{-4}\left(\frac{L}{10^{6} L_{\odot}}\right)^{3 / 4} \text {. }
$$

Because our sink particles accrete discrete Lagrangian gas cells, we apply exponential smoothing to the accretion rate for this check, with an $e$-folding time $\tau_{\text {accr }}=10^{5} \mathrm{yr}$, motivated by the fiducial timescale for massive star formation. We have experimented with setting this parameter to $10^{4}$ and $10^{6} \mathrm{yr}$ and found that it has no important effect on the SFE.

\subsubsection{IMFMED: scaling to a median value}

An alternative approach to using the IMF-averaged mean is to use the median or most likely (which are close) value over the ensemble of IMF samplings, assuming that the total stellar mass formed in the simulation can be interpreted as a coeval stellar population. Kim, Kim \& Ostriker (2016) developed this approach by sampling stellar populations with the SLUG code (da Silva, Fumagalli \& Krumholz 2012) for a range of cluster masses and deriving a fitting formula to the median value sampled at each mass scale. The median value is very small for star clusters less than a couple $100 \mathrm{M}_{\odot}$, and scales steeply towards the well-sampled value once $M_{\star} \sim 1000 \mathrm{M}_{\odot}$. This model was used in their subsequent RHD simulations (Kim et al. 2017, 2018b), and is the one we implement here.
The IMFMED model will give a value more representative of a 'typical' sampling. The disadvantage of this approach is that it lacks locality: star formation in one region of the cloud influences the amount of feedback everywhere else, which is unphysical and cannot generalize to more complicated systems in which the very definition of a progenitor cloud, and hence which stars belong to which coeval population, is ill-defined. It and IMFMEAN share another disadvantage: every sink particle has the same light-tomass ratio, which is artificially smoother than the true distribution of luminous sources in a star cluster. This motivates our next prescription.

\subsubsection{POISSON: Poisson-sampling quantized sources}

To model the discreteness of luminous sources, we can sample a quantized number of 'O-stars' in each sink particle, such that the expectation value is still the IMF-averaged value. We adopt the prescription of Su et al. (2018), which assigns to each sink particle a number of 'O-stars' sampled from a Poisson distribution, with expectation value

$\mu=\frac{m_{\text {particle }}}{\Delta m}$,

where $m_{\text {particle }}$ is the mass of the sink particle and $\Delta m$ was taken to be $100 \mathrm{M}_{\odot}$ in Su et al. (2018). Then, each 'O-star' is taken to have luminosity

$L=\Delta m\left\langle\frac{L_{\star}}{M_{\star}}\right\rangle_{\mathrm{IMF}}$.

This technique has the advantage of giving a more realistic number of feedback-injecting sources for a given amount of stellar mass. It also captures the effect of undersampling the IMF, but stochastically rather than causally as IMFmed. Although the version used in $\mathrm{Su}$ et al. (2018) sampled only one species of 'O-star', it is in principle extensible to an arbitrary number of species (Sormani et al. 2017). The details of how the luminosity is discretized, i.e. few sources versus many, is potentially important. Feedback from a single luminous source might be more efficient than that of many smaller sources because it is more concentrated and less subject to momentum cancellation (e.g. Dale 2017; Kim et al. 2018b). On the other hand, it could also be effectively weaker because luminous sources are only likely to appear once a certain amount of stellar mass has formed, at which point collapse may be more advanced and the resulting structure more difficult to disrupt. We consider two variants of this prescription, with $\Delta m=100 \mathrm{M}_{\odot}$ and $\Delta m=1000 \mathrm{M}_{\odot}$, denoted POISSON100 and POISSON1000, respectively.

A notable omission from this section is the prescription of Howard et al. (2016), which interprets each sink particle as an individual cluster, and effectively applies an IMF sampling prescription to each of these clusters individually. We have experimented with a variant of this prescription and found it to be numerically problematic in our simulation set-up, because the characteristic mass of sink particles drops as a function of mass resolution, as has generally been found in other simulations (Bate 2009; Federrath, Krumholz \& Hopkins 2017; Guszejnov et al. 2018). Thus in the limit of high resolution, feedback is made effectively weaker, and numerical convergence in the SFE is not achieved. We speculate that this type of prescription can only converge for sink particle algorithms that imprint a characteristic size or density scale other than the numerical resolution, which requires certain assumptions about the nature of star cluster formation, and indeed the very 

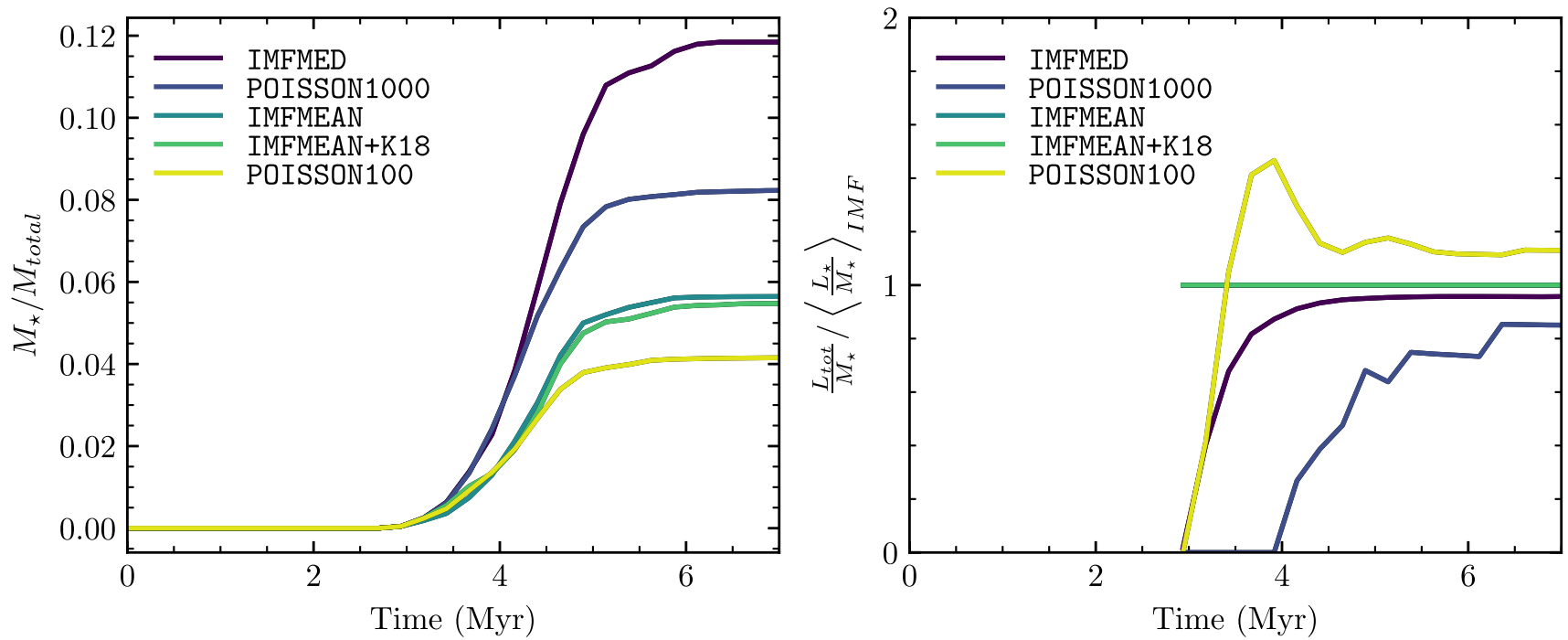

Figure 1. Left: Integrated star formation efficiency (fraction of cloud mass converted to stars) in the simulations as a function of time, for simulations run with each of the different subgrid feedback models considered (Section 2.3). Right: bulk stellar light-to-mass ratio according to the different prescriptions, normalized to the IMF-averaged value. Note that the IMFMEAN-K18 curve does not include the effect of turning off feedback according to equation (2), but we find that the effect is small (see discussion in Section 3). The different prescriptions all approach the same IMF-averaged value in the limit $M_{\star}>>10^{3} \mathrm{M}_{\odot}$, but they differ in how they approach this limit.

definition of a star cluster, that are beyond the scope of this investigation.

\section{RESULTS}

The simulated clouds evolve according to the usual sequence of events found in this type of simulation (e.g. Grudić et al. 2018a): turbulence dissipates in shocks on the crossing time-scale, and the cloud collapses into dense substructures that eventually form stars. Eventually, the cumulative effect of stellar feedback is sufficient to disrupt the cloud, halting star formation. In Fig. 1 we plot the integrated SFE $M_{\star} / M$ and the light-to-mass ratio as a function of time for each of the five different prescriptions used.

The SFE varies considerably with the feedback prescription used: IMFMED ended with an SFE of 12 percent, while POISSON100 gave 4 percent, with the others lying in between. This is despite the fact that the final light-to-mass ratios from each prescription all agreed within 10 percent, as at least $4000 \mathrm{M}_{\odot}$ forms in each simulation. We therefore find that the details of IMF sampling prescriptions for feedback can have a considerable effect on the SFE of simulated molecular clouds. In particular, we find that the IMFMEAN value of 5 per cent is reasonably consistent with Grudić et al. (2018a), which used that prescription, while IMFMED gives an SFE of 12 per cent, consistent with Kim et al. (2018b), explaining the discrepancy between those specific works.

The IMFMEAN + K18 prescription gives results that are nearly indistinguishable from the standard IMFMEAN prescription, despite the fact that it always gives less feedback. We have generally found that the fraction of time during which the criteria for turning off feedback (equation 2) are satisfied is very short compared to the lifetime of the GMC. Star particles accrete rapidly out of dense cores, and accretion halts either when the gas is exhausted or when the star particle is dynamically ejected out of its natal clump and into a void. Once equation (2) is no longer satisfied, feedback turns on and generally drives an outflow around the star. Once this outflow has been initiated, it tends not to end. Therefore even a brief lapse in the accretion rate can effectively end the accretion history.

Even assuming an infinite reservoir for accretion, an upper bound on the amount of time that equation (2) can apply can be derived from the observed properties massive stars. To maximize this time, we assume that equation (2) holds as an equality. The luminosity of stars more massive than $\sim 20 \mathrm{M}_{\odot}$ is

$\frac{L}{10^{6} L_{\odot}} \approx 0.03 \frac{M}{\mathrm{M}_{\odot}}$.

Substituting this into equation (2) gives

$\frac{\dot{M}}{\mathrm{M}_{\odot} \mathrm{yr}^{-1}}=4.7 \times 10^{-5}\left(\frac{M}{\mathrm{M}_{\odot}}\right)^{3 / 4}$.

Over the mass range of massive stars, the solution to this equation is well approximated by exponential growth with an $e$-folding time of $40 \mathrm{kyr}$, so within a few $100 \mathrm{kyr}$ the maximum stellar mass on the order of $100 \mathrm{M}_{\odot}$ must be reached. Because this is much shorter than even the shortest GMC lifetimes, the effect upon the cloudscale SFE is small. However, we emphasize that the prescription could easily have more important effects on smaller scales or shorter time-scales, such as influencing the accretion history of individual protostars or the formation of a dense star cluster.

\section{DISCUSSION}

We have shown that when simulating the evolution of an isolated molecular cloud, the specific prescription for massive stellar feedback used can affect the SFE of the cloud (and by extension, the properties of the star cluster formed) at least at the factor of $\sim 3$ level. This is despite the fact that all simulations eventually form at least several $10^{3} \mathrm{M}_{\odot}$ in stars, so the IMFs in all cases are well-sampled and the final light-to-mass ratios do not differ widely.

The simplest analytic estimate of the feedback-regulated SFE of a molecular cloud can be obtained by simply equating the bulk momentum injection rate due to feedback to the weight of the cloud 
due to self-gravity. In the limit of small SFE (Fall, Krumholz \& Matzner 2010; Murray, Quataert \& Thompson 2010; Hopkins, Quataert \& Murray 2012; Grudić et al. 2018a; Kim, Kim \& Ostriker 2018b):

$\mathrm{SFE} \propto \Sigma_{\text {gas }} / \frac{\mathrm{L}_{\star}}{\mathrm{M}_{\star}}$

where $\Sigma_{\text {gas }}$ is the mean surface density of the cloud. If this force balance is assumed to hold at the time of cloud disruption, then we would expect that the variation in SFE would not exceed the variation in $\frac{L_{\star}}{M_{\star}}$, but the simulations show that this is not the case: all simulations end with the same $\frac{L_{\star}}{M_{\star}}$ within 10 percent, yet the variation in SFE is a factor of 3.

We generally find that prescriptions that take longer to approach the fully sampled specific luminosity have SFE that can be a factor 2-3 higher than the fiducial IMFMEAN prescription. The physical reason for this is of course that the efficiency of feedback does not depend only upon the bulk ionization or momentum deposition rate: it also depends on where and when the absorption event occurs, a point deftly illustrated in recent work (Jumper \& Matzner 2018; Krumholz 2018). Specifically, recombination and cooling times are shorter at higher density, suppressing radiative heating effects, while momentum imparted in a deeper potential well provides less terminal momentum, and if the well is sufficiently deep the momentum might not be sufficient to launch a wind at all.

This raises a point that is more broadly important: the effectiveness of feedback from massive stars depends on much more than just the bulk light-to-mass ratio arising from the IMF - it depends on when and under what conditions massive stars form. This should hold quite generally, so although we have only considered schemes for injecting feedback from an assumed IMF, this has implications for calculations that attempt to resolve the IMF self-consistently. The particulars of where massive stars form in the cloud, when they form relative to other stars, and how long they take to form should all influence the behaviour of stellar feedback. The resulting influence on feedback influences the evolution of the entire cloud and the stellar population that will form.

Counterintuitively, the POISSON1000 simulation had lower SFE than the IMFMED simulation despite the fact that its light-tomass ratio was lower at all times. This implies that feedback in the POISSON1 000 was more effective for a given specific luminosity. The effect is due to the different discretizations of luminosity among the sink particles: with IMFMED, all sink particles have the same specific luminosity, while for POISSON1000 the luminosity was concentrated in only five sources at the time star formation ended. Therefore, radiative feedback from fewer, more luminous sources is more efficient, a result analogous to what has been found for the clustering of supernova explosions (Keller et al. 2014; Fielding et al. 2018). We can conjecture that the true IMF-resolved solution is probably closer to the discrete limit, because the bolometric and especially the ionizing luminosity will generally be dominated by the few most massive stars.

We note that similar experiments to those shown here were considered on a galactic scale in Su et al. (2018), who argued that galaxy-averaged quantities (e.g. stellar masses, sizes, morphologies, abundance patterns, statistics of their star formation histories) were not strongly influenced by the IMF sampling scheme. This is not surprising, as the spatial and time-scales of self-regulation via feedback in those simulations are much longer $(\gg 10 \mathrm{Myr})$, so most of the dynamics occurs in the well-sampled IMF limit (even in dwarf galaxies). Moreover other studies have shown that even artificially raising or lowering the GMC-scale SFE by much larger factors than those seen here produces relatively weak effects on galactic properties, because of global self-regulation by outflows and pressure balance in the ISM (Hopkins, Quataert \& Murray 2011; Faucher-Giguère, Quataert \& Hopkins 2013; Agertz \& Kravtsov 2015; Orr et al. 2018). However, our study here suggests that subgalactic but still large-scale quantities, e.g. properties of star clusters and lifetime/mass of molecular gas at any given time, could be significantly influenced by the physics discussed here.

It is of course possible to develop more sophisticated IMFsampling schemes (see e.g. Hu et al. 2016; Fujimoto, Krumholz \& Tachibana 2018; Emerick, Bryan \& Mac Low 2019), coupled to more detailed stellar evolution models for feedback, and this can provide some improvements for coarse-grained IMF prescriptions (especially for phenomena like SNe occurring on much longer time-scales). However, we stress that on the spatial and timescales of GMCs, this is not obviously 'more correct': the real issue is not the statistical method by which the IMF is sampled. Rather, it is the fact that these (and all of the methods discussed here) are fundamentally assigning the question of where and when massive stars form to a 'subgrid' model, which does not know about the local (resolved) conditions in the GMC/ISM. Most of the stellar mass will form wherever nature can form a $\sim 0.1 \mathrm{M}_{\odot}$ star - but low-mass cores in low-density environments almost certainly cannot form the $\gtrsim 40 \mathrm{M}_{\odot}$ stars that dominate the UV production in a massive cluster. And allowing massive stars to form 'stochastically' in such environments may likely overestimate their effects. It is also not obvious that neglecting the accretion/formation and protostellar/pre-main-sequence evolution of such stars is a valid approximation on the $\sim 1 \mathrm{Myr}$ time-scales of interest here.

As such, what we have shown is that significant, intrinsic uncertainties clearly still exist about the effects of stellar feedback at the GMC scale, at least at the level demonstrated here. These uncertainties will remain until the emergence of the IMF from GMC dynamics is understood in a self-consistent framework. Subgrid feedback prescriptions should ultimately be informed by simulations that are able to follow the formation of a stellar population at the level of resolution required to model the formation of individual massive stars in an accurate and robust manner, so that one can model in a physically motivated manner when and where in a simulation massive stars form.

\section{ACKNOWLEDGEMENTS}

We thank Mark Krumholz, Stella Offner, Claude-André FaucherGiguère, Norman Murray, Eliot Quataert, James Dale, Ian Bonnell, Hui Li, Jeong-Gyu Kim, Eve Ostriker, Michael S. Fall, Christopher Matzner, Benny Tsang, and Milos Milosavljević for enlightening discussions that informed and motivated this work. Support for MYG and PFH was provided by a James A Cullen Memorial Fellowship, an Alfred P. Sloan Research Fellowship, NSF Collaborative Research Grant \#1715847 and CAREER grant \#1455342, and NASA grants NNX15AT06G, JPL 1589742, 17-ATP17-0214. Numerical calculations were run on the Caltech compute cluster 'Wheeler,' allocations from XSEDE TG-AST130039 and PRAC NSF.1713353 supported by the NSF, and NASA HEC SMD-167592. This research has made use of the NASA Astrophysics Data System, IPYTHON (Pérez \& Granger 2007), NUMPY, SCIPY (Jones et al. 2001), NUMBA (Lam, Pitrou \& Seibert 2015), and MATPLOTLIB (Hunter 2007). 


\section{REFERENCES}

Agertz O., Kravtsov A. V., 2015, ApJ, 804, 18

Agertz O., Kravtsov A. V., Leitner S. N., Gnedin N. Y., 2013, ApJ, 770, 25

Bastian N., Covey K. R., Meyer M. R., 2010, ARA\&A, 48, 339

Bate M. R., 2009, MNRAS, 392, 1363

Baumgardt H., Kroupa P., 2007, MNRAS, 380, 1589

Bolatto A. D., Leroy A. K., Rosolowsky E., Walter F., Blitz L., 2008, ApJ, 686, 948

Colín P., Vázquez-Semadeni E., Gómez G. C., 2013, MNRAS, 435, 1701

da Silva R. L., Fumagalli M., Krumholz M., 2012, ApJ, 745, 145

Dale J. E., 2015, New Astron. Rev., 68, 1

Dale J. E., 2017, MNRAS, 467, 1067

Dale J. E., Ercolano B., Bonnell I. A., 2012, MNRAS, 424, 377

Dale J. E., Ngoumou J., Ercolano B., Bonnell I. A., 2013, MNRAS, 436, 3430

Dale J. E., Ngoumou J., Ercolano B., Bonnell I. A., 2014, MNRAS, 442, 694

Elmegreen B. G., Efremov Y. N., 1997, ApJ, 480, 235

Emerick A., Bryan G. L., Mac Low M.-M., 2019, MNRAS, 482, 1304

Fall S. M., Krumholz M. R., Matzner C. D., 2010, ApJ, 710, L142

Faucher-Giguère C.-A., Quataert E., Hopkins P. F., 2013, MNRAS, 433, 1970

Federrath C., Banerjee R., Clark P. C., Klessen R. S., 2010, ApJ, 713, 269

Federrath C., Krumholz M., Hopkins P. F., 2017, J. Phys.: Conf. Ser., 837, 1

Fielding D., Quataert E., Martizzi D., 2018, MNRAS, 481, 3325

Fujimoto Y., Krumholz M. R., Tachibana S., 2018, MNRAS, 480, 4025

Fumagalli M., da Silva R. L., Krumholz M. R., 2011, ApJ, 741, L26

Gammie C. F., Ostriker E. C., 1996, ApJ, 466, 814

Gavagnin E., Bleuler A., Rosdahl J., Teyssier R., 2017, MNRAS, 472, 4155

Grudić M. Y., Hopkins P. F., Faucher-Giguère C.-A., Quataert E., Murray N., Kereš D., 2018a, MNRAS, 475, 3511

Grudić M. Y., Guszejnov D., Hopkins P. F., Lamberts A., Boylan-Kolchin M., Murray N., Schmitz D., 2018b, MNRAS, 481, 688

Guszejnov D., Hopkins P. F., Grudić M. Y., Krumholz M. R., Federrath C., 2018, MNRAS, 480, 182

Hills J. G., 1980, ApJ, 235, 986

Hopkins P. F., 2015, MNRAS, 450, 53

Hopkins P. F., et al., 2018, MNRAS, 480, 800

Hopkins P. F., Grudić M. Y., 2019, MNRAS, 483, 4187

Hopkins P. F., Quataert E., Murray N., 2011, MNRAS, 417, 950

Hopkins P. F., Quataert E., Murray N., 2012, MNRAS, 421, 3488

Howard C. S., Pudritz R. E., Harris W. E., 2016, MNRAS, 461, 2953

Howard C. S., Pudritz R. E., Harris W. E., 2017, MNRAS, 470, 3346

Hu C.-Y., Naab T., Walch S., Glover S. C. O., Clark P. C., 2016, MNRAS, 458,3528

Hunter J. D., 2007, Comput. Sci. Eng., 9, 90
Jones E. et al., 2001, SciPy: Open source scientific tools for Python, Available at:http://www.scipy.org/

Jumper P. H., Matzner C. D., 2018, MNRAS, 480, 905

Keller B. W., Wadsley J., Benincasa S. M., Couchman H. M. P., 2014, MNRAS, 442, 3013

Kim J.-G., Kim W.-T., Ostriker E. C., 2016, ApJ, 819, 137

Kim J.-G., Kim W.-T., Ostriker E. C., Skinner M. A., 2017, ApJ, 851, 93

Kim J.-h., et al., 2018a, MNRAS, 474, 4232

Kim J.-G., Kim W.-T., Ostriker E. C., 2018b, ApJ, 859, 68

Klessen R. S., Heitsch F., Mac Low M.-M., 2000, ApJ, 535, 887

Kroupa P., 2002, Science, 295, 82

Kruijssen J. M. D., 2012, MNRAS, 426, 3008

Krumholz M. R., 2018, MNRAS, 480, 3468

Krumholz M. R. et al., 2014, in Henrik B., Ralf S. K., Cornelis P. D., Thomas H., eds, Protostars and Planets VI, University of Arizona Press, Tucson, p. 243

Krumholz M. R., Klein R. I., McKee C. F., 2011, ApJ, 740, 74

Lam S. K., Pitrou A., Seibert S., 2015, in Proceedings of the Second Workshop on the LLVM Compiler Infrastructure in HPC. LLVM '15. ACM, New York, NY, USA, p. 7

Leitherer C., et al., 1999, ApJS, 123, 3

McKee C. F., Ostriker E. C., 2007, ARA\&A, 45, 565

Murray N., Quataert E., Thompson T. A., 2010, ApJ, 709, 191

Naab T., Ostriker J. P., 2017, ARA\&A, 55, 59

Offner S. S. R., Clark P. C., Hennebelle P., Bastian N., Bate M. R., Hopkins P. F., Moraux E., Whitworth A. P., 2014, in Henrik B., Ralf S. K., Cornelis P. D., Thomas H., eds, Protostars and Planets VI, University of Arizona Press, Tucson. p. 53

Orr M. E., et al., 2018, MNRAS, 478, 3653

Pérez F., Granger B. E., 2007, Comput. Sci. Eng., 9, 21

Price D. J., Bate M. R., 2008, MNRAS, 385, 1820

Raskutti S., Ostriker E. C., Skinner M. A., 2016, ApJ, 829, 130

Rosdahl J., Teyssier R., 2015, MNRAS, 449, 4380

Skinner M. A., Ostriker E. C., 2013, ApJS, 206, 21

Skinner M. A., Ostriker E. C., 2015, ApJ, 809, 187

Sormani M. C., Treß R. G., Klessen R. S., Glover S. C. O., 2017, MNRAS, 466,407

Su K.-Y., et al., 2018, MNRAS, 480, 1666

Vázquez-Semadeni E., Colín P., Gómez G. C., Ballesteros-Paredes J., Watson A. W., 2010, ApJ, 715, 1302

Vázquez-Semadeni E., González-Samaniego A., Colín P., 2017, MNRAS, 467,1313

This paper has been typeset from a $\mathrm{T}_{\mathrm{E}} \mathrm{X} / \mathrm{L} \mathrm{A} \mathrm{E} \mathrm{X}$ file prepared by the author. 